\title{
Descriptores relacionados con el uso de recursos estilísticos para la enseñanza del español como lengua extranjera: aportaciones de una investigación en el baccalauréat francés
}

José Rienda Polo

Academia de Buenas Letras de Granada

Recibido: 25 de octubre de 2012 / Aceptado: 11 de enero de 2013

ISSN: $1697-7467$

\begin{abstract}
RESUMEN: Este artículo presenta una investigación sobre el uso de recursos estilísticos en producciones escritas en español de tres grupos de alumnos franceses de bachillerato, para comprobar si tales ítems pudieran ayudar a matizar los descriptores de los distintos niveles de competencia del MCERL. Los resultados apuntan a una relación directa entre nivel de competencia y uso de recursos de estilo y, por lo tanto, a la validez de estos como descriptores que pueden ayudar a precisar la caracterización de la competencia de los aprendices en la L2.

Palabras clave: competencia comunicativa, composición escrita, recursos estilísticos, español lengua extranjera, descriptores de niveles de competencia.
\end{abstract}

Descriptors Related to Stilistyc Devices in Spanish as a Foreign Language: A Contribution from a Research in the French Baccalauréat

\begin{abstract}
In this paper we analyze the use of stylistic resources in the written language samples of three French high school groups of learners of Spanish to check whether such items could help clarify the descriptors for the CEFRL competence levels. Results show a direct relationship between competence level and use of style resources; thus suggesting the validity of such descriptors to precise the characterization of L2 learners' competences.

Keywords: communicative competence, writing, stilistyc devices, Spanish as a foreign language, descriptors for competence levels
\end{abstract}

\section{Marco teórico}

Es evidente que los actuales enfoques y procesos didácticos para la enseñanza del español como lengua extranjera (ELE/EL2) se ajustan a los niveles de referencia establecidos en el Marco Común Europeo de Referencia para las Lenguas, y responden en gran medida a los presupuestos metodológicos que de él derivan, concretándose en la práctica de las aulas a partir de propuestas como la amparada en los llamados escenarios de comunicación, delimitada y estudiada por autores como González Piñeiro, Guillén y Vez (2010), entre otros. Con este enfoque "accional" se entreteje el enfoque competencial, según el cual la 
finalidad de la enseñanza y el aprendizaje de una lengua es lograr un aumento progresivo de la competencia comunicativa ${ }^{1}$.

Recordemos que el enfoque de la formación basada en competencias $(F B C)$ fue iniciado en EE.UU. durante los años 20 del pasado siglo a partir de ciertas reformas educativas requeridas por los sectores industriales y comerciales del momento (Blas, 2008), mientras su integración en la reforma de la formación del profesorado de dicho país tuvo lugar ya en los últimos años de la década de los sesenta (Tuxworth, 1989). Dicha orientación ha sido finalmente trasladada a la educación obligatoria y homogeneizada para el Espacio Europeo de Educación Superior con la Declaración de Bolonia de 1999.

Esta alusión al origen y desarrollo de la $F B C$ y su prolongación hacia la formación competencial generalizada, resulta obviamente necesaria para la fijación del concepto de competencia comunicativa que precisamos, constatando siempre que la idea de competencia difiere ya bastante de lo designado en su origen (Fleming, 2006: 2):

The intention was to place the primary emphasis on outputs (an account of the specific occupational role broken down into performance statements) and to recognise that the particular inputs (training courses, work experience, prior learning) were contingent; it matteered more what prospective employees could actually do, not what training they had attended.

A pesar de todo, una cuestión que llama especialmente la atención es el hecho de que, si bien es cierto que a finales del siglo pasado adquirió cierta importancia la significatividad de los procesos de aprendizaje (Sánchez Pérez, 1993; Madrid y McLaren, 1995), en el ámbito de la enseñanza de lenguas extranjeras, por el contrario, se mantiene la importancia del resultado, es decir, el valor determinante del producto final del proceso de aprendizaje (Madrid, 1997) como índice o descriptor de gradación. Como la evaluación es una actividad curricular programada, es evidente que todo diseño curricular centrado en el producto incluye igualmente una evaluación centrada en el producto, según ejemplifica Madrid (1997) a través de los programas gramático-funcionales de los sesenta (cfr. Brooks, 1966; Rivers, 1968) o los de carácter nocional-funcional de los ochenta (Wilkins, 1976; Johnson, 1989).

Es decir, se trata de una evaluación "previamente planificada y programada de acuerdo con los objetivos del programa (syllabus) [...] y encaminada hacia la comprobación de esos objetivos finales de enseñanza y aprendizaje y diseñada de acuerdo con esos criterios previamente establecidos (criterion referenced)." (Madrid, 1997: 253).

Por otro lado, no resultaría complicado en exceso coincidir en que la lectura y conocimiento de los textos literarios así como la práctica de la escritura de intención literaria contribuyen al desarrollo del alumno en el marco no ya solo de la enseñanza de lenguas extranjeras o de la propia competencia comunicativa, sino también en el espacio genérico de la inserción cultural y la formación como ciudadanos. En este sentido, el concepto de competencia comunicativa funciona como un concepto global que abarca subcompetencias

\footnotetext{
${ }^{1}$ En relación con esta se sitúa la competencia literaria, cuyo desarrollo vinculado a los procesos didácticos continúa en su irónicamente ilustre pervivencia como adjudicatario del rol, siempre secundario, que supone su consideración como recurso válido para la mejora de la competencia comunicativa (Rienda, 2010). Sin embargo, la integración de la competencia literaria en el marco general de la competencia comunicativa por un lado, y, por otro, la vinculación de los términos competencia y literatura nos lleva, junto a otros autores (Olsbu y Salkjelsvik, 2006), a considerar una problemática conceptual que se acrecienta, aún más si cabe, en el ámbito de la enseñanza de ELE.
} 
específicas (Canale y Swain, 1996; MCERL, 2002²) -entre ellas la literaria-sin que esto signifique que estas últimas han de entenderse como subordinadas o como de menor importancia, pues han de ser tratadas en su especificidad para alcanzarlas siempre al amparo del enfoque comunicativo en su doble vertiente funcional y significativa (Núñez, 2003: 72) que es irrenunciable para superar el gramaticalismo tradicional:

Los enfoques basados en presupuestos funcionalistas y comunicativos [...] en general, han aparejado una evolución [...] en el sentido de que la atención ha ido pasando de la lengua como objeto de conocimiento al uso o funcionalidad de la misma.

Así pues, entendida la competencia comunicativa como el conjunto de estrategias, instrumentos y recursos que conforman el conocimiento que necesita el individuo para la optimización de su actividad comunicativa, podemos asimismo conceptuar la competencia literaria amparados en la teorización que en su momento realizó Rienda (2010: 54):

[...] la adquisición sociocultural del intertexto ${ }^{3}$ determinado por la involucración del receptor, así como de la perfectibilidad de la producción cognitivo-intencional de la especificidad literaria, adquisición desarrollada en acto y correlacionada con el horizonte de competencias genérico-instrumentales tanto para la optimización de los mensajes en el marco de la competencia comunicativa como para la culminación del singular proceso didáctico de la literatura en su triple dimensión ontológica, social-axiológica y academicista.

Otro de los elementos que sustentan las bases teóricas de esta investigación tiene que ver con la destreza de la expresión escrita. Nuestro trabajo se centra en la composición de textos escritos de intención literaria con arreglo a consignas para la escritura creativa (Rodari, 1983; Alvarado, Rodríguez y Tobelem, 1981; Samoilovich, 1979; Freinet, 1973) en un aula de español como lengua extranjera, y sobre todo en el uso por parte de los sujetos de recursos estilísticos cuya aparición suele verse mermada debido, por un lado, a la falta de dominio de la lengua meta y, por otro, a la preocupación por la corrección gramatical y ortográfica, aspectos que en modo alguno hay que descuidar en la enseñanza escolar de las lenguas, pero que -como afirma Bronckart (2008: 12) - "deben ser concebidas y enseñadas como apoyo conceptual al servicio de objetivos de dominio práctico; lo que implica que deberían ser reformuladas en una perspectiva funcional y textual". Por estos motivos, un factor de peso para conseguir la mejora de la expresión escrita en estudiantes de L2 radica en la necesidad de introducir cambios en las rutinas didácticas de composición del texto y en su posterior evaluación.

El modelo de proceso por el que nos hemos decantado como explicativo del funcionamiento de la composición escrita, y para realizar la correspondiente propuesta didáctica,

${ }^{2}$ El MCERL establece, por ejemplo, tres subcompetencias -lingüística, sociolingüística y pragmática- que a su vez se componen de otras más concretas.

${ }^{3} \mathrm{El}$ intertexto, recordamos, es (Mendoza, 1999: 18):

[...] el esencial conjunto de saberes, estrategias y de recursos lingüístico-culturales que se activan a través de la recepción literaria para establecer asociaciones de carácter metaliterario e intertextual y que permiten la construcción de conocimientos significativos de carácter lingüístico y literario que se integran en el marco de la competencia literaria. 
arranca de los trabajos de Flower y Hayes (1980), difundidos en España sobre todo por Cassany (1989, 1993 y 1995), que distinguen dos tipos de escritura: "prosa de escritor" y "prosa de lector"; la primera -que es la que se ha practicado en nuestro estudio- remite a la expresión libre de sentimientos, y la segunda, más elaborada, a la preocupación por que el lector comprenda el texto. Escribir se concibe como una habilidad cognitivo-lingüística muy compleja que se produce en tres pasos o fases recurrentes: planificación, textualización y revisión.

A partir de aquí, la combinación de aspectos teóricos referidos hasta ahora determinan que, para los fines de nuestra investigación, los objetivos de aprendizaje que se han concretado para el aula maticen e integren a) objetivos de carácter instrumental para el desarrollo de la competencia comunicativa en la enseñanza de lenguas extranjeras, b) objetivos relativos al uso de recursos estilísticos vinculados a la competencia comunicativa escrita en la enseñanza de lenguas extranjeras, y c) objetivos más específicos de creatividad en la escritura de intención literaria (Núñez y Romero, 2003; Rienda, 2010). En esta determinación se ha contemplado asimismo el hecho de que casi tres lustros después de la propuesta de reforma del Espacio Europeo de Educación Superior, también tienden a converger, al menos en los aspectos terminológicos en lo que a la $\mathrm{FBC}$ se refiere, las enseñanzas medias de los distintos países integrados en el Plan Bolonia ${ }^{4}$, pues de lo que se trata es, en líneas generales, de entender el currículum como un instrumento de cambio e innovación en el que los procesos de enseñanza no se disocian de los procesos de aprendizaje y donde los proyectos curriculares se encaminan no solo al desarrollo del conocimiento, sino también a cómo se produce dicho desarrollo (Navarro, 1992), siendo destacables tres características en función de sus implicaciones didácticas: el carácter abierto del currículum (García Rivera, 1995), la dimensión globalizadora e integradora y, en tercer lugar, la integración de la teoría genética del aprendizaje, la teoría del aprendizaje verbal significativo de Ausubel (1968) y la teoría esquemática de la estructura cognitiva del sujeto.

En definitiva, si lo que nos interesa desde el concepto de competencia como referencia es, precisamente, lo que sabe hacer el alumno con los conocimientos que ha adquirido (Olsbu y Salkjelsvik, 2006) -sin soslayar en ningún momento la dificultad añadida que supone la evaluación no ya de la adquisición de destrezas, sino también de la instrumentalización que de ellas realiza-, surge la cuestión nodal que presentamos: considerando que la competencia literaria "es sinónimo de una serie de encuentros en los que se ven involucrados tanto la cultura-meta, el autor, la experiencia personal del alumno y la influencia del profesor" (Olsbu y Salkjelsvik, 2006: 4), ¿cómo se podría cuantificar, sponderar, medir, o, en definitiva, evaluar el resultado de tales encuentros? O, más concreto aún, “¿qué estándares textuales se activan y se fomentan en el alumno al trabajar con un texto literario" (id.) y qué elementos de los asociados tradicionalmente al lenguaje literario, como son los recursos estilísticos, se amplifican o minimizan privativamente en el ámbito de la enseñanza de ELE mediante la composición de textos escritos? Sobre estas cuestiones se centra el presente trabajo para establecer un corpus de descriptores relativos con el uso apropiados que determinen los

${ }^{4}$ En este sentido, destacamos el caso de la educación secundaria y el bachillerato franceses, espacio en el que hemos desarrollado nuestra investigación y que analizamos más adelante. 
logros para la gradación de los niveles A2/B1+ a partir de los mencionados elementos de amplificación o minimización privativas de la competencia literaria.

\section{Objetivos e hipótesis}

La hipótesis de partida de la investigación que aquí se presenta puede formularse en los siguientes términos: en el proceso de adquisición de la competencia comunicativa en ELE, los recursos estilísticos aumentan su presencia progresivamente, por lo que podrían ser válidos y útiles como descriptores para la detallar y concretar los niveles A2/B1+ competencia en la enseñanza y el aprendizaje del español como lengua extranjera.

En consonancia con esta hipótesis, los objetivos planteados son los siguientes:

1. Detectar la presencia de recursos estilísticos en producciones de escritura creativa de estudiantes de español como lengua extranjera.

2. Determinar qué recursos aparecen y con qué frecuencia lo hacen.

3. Establecer, mediante la marca de presencia/ausencia de dichos elementos en la composición de textos escritos, un gradiente de dominio para los niveles A2/B1+ que pueda ser utilizado para una evaluación más precisa de la competencia de los estudiantes de ELE.

\section{Diseño Y Elementos PRinCipales de LA inVestigación}

\subsection{Tipo}

En función de las características generales que conforman nuestra propuesta de investigación tal y como han quedado reflejadas en la hipótesis y objetivos de partida, se ha optado por un diseño de investigación cuantitativa de tipo descriptivo comparativo. El diseño es cuasiexperimental (Dendaluce, 1994), como suele ser habitual en la investigación educativa, pues se estudia un contexto real donde tanto las características de los programas como los grupos están definidos de antemano por la institución. En esquema, el diseño puede sintetizarse en el siguiente diagrama:

$$
\begin{array}{lll}
\mathrm{M} 1 \leftrightarrow & \mathrm{O} 1 & \\
\mathrm{M} 2 \leftrightarrow & \mathrm{O} 2 & \mathrm{O} 1(=/ \neq / \approx) \mathrm{O} 2 \\
\mathrm{M} 3 \leftrightarrow & \mathrm{O} 3 & \mathrm{O} 1(=/ \neq / \approx) \mathrm{O} 2(=/ \neq / \approx) \mathrm{O} 3 \\
\mathrm{Mn} \leftrightarrow & \mathrm{On} & \mathrm{O} 1(=/ \neq / \approx) \mathrm{O} 2(=/ \neq / \approx) \mathrm{O} 3(=/ \neq / \approx) \mathrm{On}
\end{array}
$$

Donde:

- M1, M2, M3, Mn..., representan a cada uno de los grupos de sujetos estudiados;

- O1, O2, O3, On... representan la información (observaciones) recogida en cada grupo.

- O1 a On..., parte lateral, indican las comparaciones que se llevan a cabo entre cada una de las muestras, cuyos resultados pueden ser iguales $(=)$, diferentes $(\neq)$, o semejantes $(\approx)$ ( $c f r$. Rivas, 1991 y 2009). 


\subsection{Contexto del estudio y sujetos experimentales}

El estudio empírico de este trabajo se ha realizado en dos institutos de Le Havre (Normandía, Francia). Ambos centros son Institutos de Enseñanza Técnica y Profesional. El Lycée des Métiers Jules Lecesne (Académie de Rouen) es uno de los institutos públicos más importantes de la región de Haute Normandie. El aprendizaje de una segunda lengua extranjera (español o alemán) se incluye en las formaciones de Baccalauréat Professionnel (Bac pro): Commerce, Hotellerie et Mode y Baccalauréat Technologique: Hotellerie. Ambos bachilleratos se cursan en tres años. El Lycée Germaine Coty es un instituto privado gestionado por la enseñanza católica (Enseignement Catholique de l'Académie de Rouen). En él se imparten igualmente tanto bachilleratos técnicos como profesionalizadores. En nuestro caso, se ha escogido un bachillerato de ciencias y tecnologías de laboratorio (Bac STL), modalidad que contempla la enseñanza de una segunda lengua extranjera como materia optativa ${ }^{5}$.

Estos alumnos se preparan para superar unas pruebas orales y escritas al final del bachillerato en las que se incluye la asignatura Espagnol Langue Vivante $2^{6}$. Estas pruebas difieren entre la modalidad de Bac pro y la de Bac techno dado que las diferencias de nivel también son significativas entre ambas modalidades, oscila entre el coeficiente 2 y el coeficiente $5^{7}$, siendo más elevada la capacitación entre los alumnos de Bac techno.

La normativa vigente en materia de educación ( L'Education Nationale) establece cinco competencias básicas en materia de enseñanza y aprendizaje de lenguas extranjeras en la educación superior (Collège y Lycée). Dos de las cinco competencias son exclusivamente orales: "expresión fuida" (parler en continu) e "interacción oral" (reagir et dialoguer) ${ }^{8}$. Dependiendo del nivel los contenidos y objetivos previstos de cara a la superación del bachillerato son:

1. Coeficiente 2. Baccalauréat économique et social (TES): bachillerato de ciencias sociales; Baccalauréat scientifique (TS): bachillerato de ciencias; Baccalauréat sciences et technologies de la gestion (TSTG): bachillerato de ciencias y tecnología de la gestión.

- Fluidez expresiva:

* Expresarse de manera simple acerca de diversos temas.

* Tomar la palabra en público.

* A partir de unas notas previas, reproducir un texto con sus propias palabras.

* Relatar experiencias vividas, aportando datos, ejemplos, etc.

* Explicar un proyecto, un proceso, precisar un modo de empleo.

* Expresar sentimientos y opiniones personales.

* Argumentar para convencer.

\footnotetext{
${ }^{5}$ A partir del curso 2012-2013, esta materia adopta el carácter de obligatoria.

${ }^{6}$ Para un mejor conocimiento del contexto curricular y de sus condicionamientos sobre la investigación, cabe reseñar que en Francia se exige a los docentes el establecimiento coordinado de los objetivos mínimos para cada grupo-clase respecto a la competencia escrita en lengua extranjera; se impone, además, la utilización de la gramática únicamente como herramienta auxiliar para el desarrollo comunicativo, evitando la impartición de clases magistrales de gramática y eximiendo al alumnado de la obligación de su estudio: únicamente se recurre a los manuales de gramática si los estudiantes lo solicitan con el fin de solucionar dudas.

${ }^{7}$ Únicamente en alumnos que cursan la modalidad de bachillerato literario y que han elegido como asignatura optativa Langue approffondie.

${ }^{8}$ Cfr. Bulletin Officiel Spécial de l'Education Nationale 2011-3.
} 
- Interacción oral:

* Hacer frente a diversas situaciones, articulando y pronunciando correctamente.

* Mantener una conversación y el contacto para: intercambiar información, reaccionar ante los sentimientos y expresar un punto de vista u opinión.

* Tomar parte en una discusión para explicar, comentar, comparar y argumentar a favor o en contra.

* Entrevistar y ser entrevistado, conducir una entrevista previamente preparada y tomar la iniciativa.

* Realizar peticiones.

2. Coeficiente 4. Baccalauréat litteraire (TL): bachillerato de humanidades.

- Fluidez expresiva:

* Expresarse de forma detallada y organizada a partir de una amplia variedad de temas relativos a campos de interés o de conocimiento.

* Separarse espontáneamente de un texto preparado y reaccionar a partir de temas relacionados propuestos por el profesor o los compañeros.

* Exponer un tema de forma clara y metódica haciendo hincapié en los aspectos significativos.

* Hacer una descripción clara y detallada sobre un tema preparado.

* Expresar con precisión los sentimientos y las opiniones personales.

* Argumentar de forma clara y concisa.

- Interacción oral:

* Participar en conversaciones largas con nativos de la lengua.

* Proponer un tema, dirigir una conversación y terminarla.

* Intercambiar y solicitar informaciones precisas.

* Tomar parte en una conversación sobre temas conocidos o estudiados: exponer puntos de vista, evaluar los puntos de vista de los demás, emitir hipótesis.

* Desarrollar ideas y opiniones de forma precisa a partir de argumentos sobre temas complejos.

* Argumentar y reaccionar ante los argumentos de los demás.

* Formular preguntas, exponiendo las causas y las consecuencias, las ventajas y los inconvenientes.

* Entrevistar y ser entrevistado. Conducir una entrevista con eficacidad, bienestar y autonomía.

* Señalar y corregir lapsus y errores.

Con respecto a los sujetos experimentales, cuentan con un nivel similar en Espagnol Langue Vivante 2. Variables como el sexo o la edad no se han tenido en cuenta, al no tener relevancia para este estudio. La determinación del nivel de dominio de español, que es la variable esencial, fue establecida por el profesorado del centro al comienzo del curso escolar mediante las correspondientes pruebas homologadas 9 . La muestra puede considerarse ecológica en tanto que extensible a la diversidad del alumnado. Respecto a la distribución de los sujetos de la muestra, concretamos que 9 se encuandran en el nivel A2, 10 en el B1 y 10 en el B1+.

${ }^{9}$ La profesora Carmen Aguilera Tapiador certificó para nosotros los mencionados niveles y garantizó la concreción correcta de esta contextualización. 


\subsection{Instrumentos y procedimiento de instrucción}

El Cuaderno de composición escrita (Rienda, 2006) es el instrumento en torno al cual se han articulado las actividades y la investigación empírica. Se trata de un material de trabajo sobre el que se han realizado dos investigaciones previas que avalan su validez. Así, en 2006 ya determinamos (Rienda, 2006: 7-15), a parir de una selección de veinte actividades de composición escrita tomadas de cuatro fuentes distintas (Rodari, 1983; Alvarado, Rodríguez y Tobelem, 1981; Samoilovich, 1979 y Freinet, 1973), las dificultades tipo para cada una de ellas, las microdestrezas de la composición escrita que estas trabajan particularmente y, además de otros descriptores, los aspectos de la competencia comunicativa que se desarrollan especialmente en cada caso y que han servido de variables: la hipótesis fantástica, el cuestionario disparatado, el muro descascarado, la chistera del mago, comicar, traducción imaginaria, ensalada de fábulas, palabras robadas, principio dado, terminar desde la mitad, el binomio fantástico, adjetivar, el cliente y sus pleonasmos, composición dirigida, fábulas en clave obligada, con nombre propio, confusión de cuentos, acróstico, topograma y texto libre fueron los títulos de las actividades examinadas mediante muestreo y análisis en varios centros educativos de la provincia de Granada (España) durante el curso 2005/2006.

Por otro lado, en 2011 se llevó a cabo otro estudio que utilizó el mencionado Cuaderno de composición escrita, el cual evaluó la incidencia funcional de su aplicación como propuesta didáctica, durante un semestre, en las aulas de educación primaria. En dicho estudio se analizó la variación competencial del alumnado respecto a los estándares textuales de adecuación, corrección, coherencia y cohesión en la producción de textos escritos por parte de un grupo experimental frente a un grupo control tras la finalización del Cuaderno de composición escrita como variable independiente. Los resultados avalaron la funcionalidad del instrumento con los siguientes resultados cuantificados de 0 a 5 , donde 0 es nivel bajo y 5 nivel alto (Alarcón, 2011: 122).

Tabla 1: Resultados obtenidos en un estudio previo con el instrumento utilizado.

\begin{tabular}{|c|c|c|c|c|}
\hline & COHERENCIA & COHESIÓN & ADECUACIÓN & CORRECCIÓN \\
\hline GRUPO CONTROL & 4,42 & 4,22 & 3,76 & 3,54 \\
\hline $\begin{array}{c}\text { GRUPO } \\
\text { EXPERIMENTAL }\end{array}$ & 3,76 & 3,09 & 3,05 & 2,94 \\
\hline
\end{tabular}

Los criterios aplicados en la selección de actividades para el proceso de instrucción de esta investigación atienden, en primer lugar, a la viabilidad que ofrecen para el desarrollo de la composición escrita en los niveles de logro lingüístico que se definen en los niveles de referencia considerados y, en segundo lugar, al hecho de que, al ser propuestas de escritura creativa y de intención literaria, presumiblemente implican el uso de recursos estilísticos.

Las actividades elegidas para nuestro estudio en aulas de ELE del bachillerato francés responden a los siguientes objetivos didácticos: 
Estrategia: ENSALADA DE FÁBULAS ( $E N)$.

Fuente: Gramática de la fantasía.

Tipo de técnica: Creativa (prosa).

Objetivos: - Conocimiento de la literatura de tradición popular.

- Estimulación de la capacidad creativa e imaginativa.

- Ordenamiento lógico.

- Creación de textos en prosa.

Estrategia: EL CUESTIONARIO DISPARATADO $(C U)$.

Fuente: Teoría y práctica de un taller de escritura.

Tipo de técnica: Creativa (prosa).

Objetivos: - Desarrollo de la competencia léxica.

- Estimulación de la capacidad creativa e imaginativa.

- Práctica de la imitatio de textos literarios.

Estrategia: FÁBULAS EN CLAVE OBLIGADA (FA).

Fuente: Gramática de la fantasía.

Tipo de técnica: Creativa (prosa).

Objetivos: - Conocimiento de la literatura de tradición popular.

- Estimulación de la capacidad creativa e imaginativa.

- Ordenamiento lógico.

- Creación de textos imitando las características de las narraciones populares.

Estrategia: LA CHISTERA DEL MAGO $(\mathrm{CH})$.

Fuente: Cómo jugar y divertirse con escritores famosos.

Tipo de técnica: Creativa (prosa).

Objetivos: - Activación de la consciencia ortográfica.

- Estimulación de la capacidad creativa e imaginativa.

- Adquisición de mecanismos de coherencia y cohesión textual.

- Imitatio de textos en prosa de escritores famosos.

Estrategia: TOPOGRAMA (TO).

Fuente: Gramática de la fantasía.

Tipo de técnica: Creativa (poesía).

Objetivos: - Conocimiento de las especificidades de la creación poética.

- Estimulación de la capacidad creativa e imaginativa.

- Escritura de textos poéticos.

Estrategia: ACRÓSTICO $(A C)$.

Fuente: -

Tipo de técnica: Creativa (poesía).

Objetivos: - Conocimiento de las especificidades de la creación poética.

- Estimulación de la capacidad creativa e imaginativa.

- Escritura de acrósticos como técnica de creación poética. 
Por otro lado, para el registro cuantificado de los resultados de cada uno de los aspectos estudiados se han utilizado listas de control que incluían un repertorio alfabético de los recursos literarios considerados.

A los efectos pretendidos en este estudio cabe precisar: primero, los recursos literarios se entienden como alteraciones o variaciones del lenguaje corriente realizadas conscientemente por el autor; segundo, los recursos literarios no son propios de un género literario en particular, sino del lenguaje literario o de intención estética en general en tanto que puede entenderse como artificio creativo; y tercero, conscientes de la dificultad de establecer los límites a la división tradicional de las figuras retóricas entre figuras de pensamiento y figuras de lenguaje, se ha prescindido de tal clasificación, optando por un orden alfabético.

Por último, se indica la correspondencia entre los niveles marcando en negrita los referenciados en el apartado de la contextualización, y se determina como punto de partida la tabla de "Niveles comunes de referencia: escala global" del Marco Común Europeo de Referencia para las Lenguas (2002: 26): A1 (nivel principiante); A2/A2+ (nivel elemental o básico); B1 (Nivel preintermedio); B1+ (nivel intermedio); B2 (nivel intermedio alto); C1 (nivel avanzado); C2 (nivel muy avanzado).

\section{Datos obtenidos}

La herramienta utilizada para el análisis estadístico ha sido el programa SPSS en su versión 20.0.0. La fiabilidad y validez obtenidas se catalogan como buena y significativa según las referencias del Alpha de Cronbach y el coeficiente de correlación de Pearson. Por otro lado, se ha utilizado el modelo de efectos fijos de análisis de la varianza en tanto que sólo se persigue el análisis de la presencia del recurso literario.

Los principales datos obtenidos en relación con el uso de figuras estilísticas en las producciones de los sujetos se ofrecen a continuación en las tablas 2 a 8 considerados según diversos factores: curso/grupo (tabla 2), niveles de competencia (tabla 3), actividades realizadas (tablas 4 y 5), y sujetos participantes en cada grupo (tablas 6, 7 y 8). Figuras contempladas en la planificación de nuestro estudio, pero no usadas por ninguno de los sujetos han sido: alegoría, aliteración, anacoluto, asíndeton, calambur, comunicación, gradación, juego de palabras, metonimia, paradoja, paronomasia, perífrases, pleonasmo, quiasmo, reticencia, silepsis, sinécdoque, tropos y zeugma. 
Tabla 2: Listado de figuras y aparición de las mismas en las composiciones escritas de cada uno de los grupos estudiados.

\begin{tabular}{|c|c|c|c|c|c|}
\hline \multirow[b]{2}{*}{$\begin{array}{l}\text { FIGURA } \\
\text { POÉTICA }\end{array}$} & \multicolumn{5}{|c|}{ GRUPO } \\
\hline & $\begin{array}{l}\text { BAC } \\
\text { PRO }\end{array}$ & $\begin{array}{c}\text { BAC } \\
\text { TECHNO }\end{array}$ & $\begin{array}{c}\text { PREMIER } \\
\text { STL }\end{array}$ & $\begin{array}{c}\text { SECONDE } \\
\text { BAC } \\
\text { TECHNO } \\
\text { HÔTELLERIE }\end{array}$ & TERMINALE \\
\hline Anadiplosis & o & o & o & o & + \\
\hline Anáfora & $\mathrm{o}$ & o & $\mathrm{o}$ & + & + \\
\hline Antítesis o contraste & o & o & o & + & + \\
\hline Antonomasia & o & + & + & + & + \\
\hline $\begin{array}{l}\text { Apóstrofe o } \\
\text { exclamación }\end{array}$ & + & + & + & + & + \\
\hline Epanadiplosis & o & o & o & o & + \\
\hline Epifonema & o & + & + & + & + \\
\hline Epíteto & o & + & + & + & + \\
\hline Hipérbaton & o & o & o & o & + \\
\hline Hipérbole & o & o & o & + & + \\
\hline Imagen & o & o & o & + & + \\
\hline Interrogación retórica & o & o & o & o & + \\
\hline Ironía & o & o & o & o & + \\
\hline Lítotes o atenuación & o & o & o & o & + \\
\hline Metáfora & o & + & + & + & + \\
\hline Onomatopeya & + & + & + & + & + \\
\hline Paralelismo & + & + & + & + & + \\
\hline Polisíndeton & o & o & o & o & + \\
\hline Prosopopeya & o & + & + & + & + \\
\hline Sarcasmo & o & o & o & o & + \\
\hline Símbolo & o & o & o & + & + \\
\hline Símil o comparación & + & + & + & + & + \\
\hline
\end{tabular}

Presencia: + Ausencia: o

Como se puede apreciar, solo hay cuatro recursos $(1,64 \%)$ que aparecen en todos los grupos, la apóstrofe, la onomatopeya el paralelismo y el símil. Otras figuras, como la antonomasia, el epifonema, el epíteto, la metáfora y la prosopopeya, aparecen en todos los grupos $(2,05 \%)$, menos en el Bac Pro, el de nivel más bajo de dominio. Cinco figuras $(2,05 \%)$ aparecen solo en los dos grupos más avanzados (anáfora, antítesis, hipérbole, imagen y símbolo); limitan su presencia al último grupo la anadiplosis, la epanadiplosis, el hipérbaton, la interrogación retórica, la lítotes, el polisíndeton y el sarcasmo (7, un 2,87\%), mientras que un total de $18(7,38 \%)$ no aparece en ninguno de los grupos.

$\mathrm{Si}$ atendemos ahora a los niveles de competencia de los del MCERL considerados en el estudio, la distribución del uso de figuras en cada uno queda reflejada en la tabla 3. 
Tabla 3: Listado de figuras y uso de las mimas en las composiciones escritas de cada uno de los grupos estudiados

\begin{tabular}{|c|c|c|c|}
\hline \multirow[b]{2}{*}{$\begin{array}{l}\text { FIGURA } \\
\text { POÉTICA }\end{array}$} & \multicolumn{3}{|c|}{ NIVELES DE COMPETENCIA } \\
\hline & $\begin{array}{l}\text { ELEMENTAL } \\
\text { O BÁSICO } \\
\text { A2/A2+ }\end{array}$ & $\begin{array}{l}\text { PREINTERMEDIO } \\
\text { B1 }\end{array}$ & $\begin{array}{c}\text { INTERMEDIO } \\
\text { B1+ }\end{array}$ \\
\hline Anadiplosis & $\mathrm{O}$ & o & + \\
\hline Anáfora & $\mathrm{O}$ & + & + \\
\hline Antítesis o contraste & o & + & + \\
\hline Antonomasia & + & + & + \\
\hline Apóstrofe o exclamación & + & + & + \\
\hline Epanadiplosis & o & o & + \\
\hline Epifonema & + & + & + \\
\hline Epíteto & + & + & + \\
\hline Hipérbaton & o & o & + \\
\hline Hipérbole & $\mathrm{o}$ & + & + \\
\hline Imagen & o & + & + \\
\hline Interrogación retórica & $\mathrm{o}$ & $\mathrm{o}$ & + \\
\hline Ironía & o & o & + \\
\hline Lítotes o atenuación & $\mathrm{o}$ & $\mathrm{o}$ & + \\
\hline Metáfora & + & + & + \\
\hline Onomatopeya & + & + & + \\
\hline Paralelismo & + & + & + \\
\hline Polisíndeton & o & o & + \\
\hline Prosopopeya & + & + & + \\
\hline Sarcasmo & o & o & + \\
\hline Símbolo & 0 & + & + \\
\hline Símil o comparación & + & + & + \\
\hline
\end{tabular}

Presencia: + Ausencia: o

En el nivel inicial de los que estamos considerando, el A1/A2+, se usan 9 figuras (3,69\%); en el siguiente nivel, correspondiente al B1 de MCERL, aparecen 14 (5,74\%); en el último de los estudiados, el $\mathrm{B} 1+$, las producciones de los estudiantes incluyen un total de $22(9,02 \%)$.

Considerando ahora las actividades que se utilizaron en el periodo de instrucción, los resultados son los que se presentan en las tablas 4 y 5 . 
Tabla 4: Listado de figuras y uso de las mismas en las composiciones escritas producidas en cada una de las actividades realizadas

\begin{tabular}{|c|c|c|c|c|c|c|}
\hline \multirow[b]{2}{*}{$\begin{array}{l}\text { FIGURA } \\
\text { POÉTICA }\end{array}$} & \multicolumn{6}{|c|}{ ACTIVIDADES } \\
\hline & $\begin{array}{c}\text { Ensalada } \\
\text { de } \\
\text { fábulas }\end{array}$ & $\begin{array}{c}\text { El } \\
\text { cuestionario } \\
\text { disparatado }\end{array}$ & $\begin{array}{c}\text { Fábulas } \\
\text { en clave } \\
\text { obligada }\end{array}$ & $\begin{array}{c}\text { La } \\
\text { chistera } \\
\text { del mago }\end{array}$ & Topograma & Acróstico \\
\hline Anadiplosis & $\mathrm{o}$ & $\mathrm{o}$ & $\mathrm{o}$ & $\mathrm{o}$ & + & + \\
\hline Anáfora & $\mathrm{o}$ & $\mathrm{o}$ & $\mathrm{o}$ & $\mathrm{o}$ & + & $\mathrm{o}$ \\
\hline $\begin{array}{l}\text { Antítesis o } \\
\text { contraste }\end{array}$ & $\mathrm{o}$ & + & $\mathrm{o}$ & $\mathrm{o}$ & + & o \\
\hline Antonomasia & $\mathrm{o}$ & + & + & o & o & o \\
\hline $\begin{array}{l}\text { Apóstrofe o } \\
\text { exclamación }\end{array}$ & + & o & + & + & + & o \\
\hline Epanadiplosis & $\mathrm{o}$ & $\mathrm{o}$ & $\mathrm{o}$ & $\mathrm{o}$ & $\mathrm{o}$ & + \\
\hline Epifonema & $\mathrm{o}$ & $\mathrm{o}$ & + & + & $\mathrm{o}$ & $\mathrm{o}$ \\
\hline Epíteto & + & + & + & + & + & + \\
\hline Hipérbaton & $\mathrm{o}$ & $\mathrm{o}$ & $\mathrm{o}$ & o & $\mathrm{o}$ & + \\
\hline Hipérbole & + & + & + & + & + & $\mathrm{o}$ \\
\hline Imagen & $\mathrm{o}$ & + & $\mathrm{o}$ & $\mathrm{o}$ & + & + \\
\hline $\begin{array}{l}\text { Interrogación } \\
\text { retórica }\end{array}$ & $\mathrm{o}$ & $\mathrm{o}$ & $\mathrm{o}$ & + & o & o \\
\hline Ironía & 0 & + & 0 & o & $\mathrm{o}$ & $\mathrm{o}$ \\
\hline $\begin{array}{l}\text { Lítotes o } \\
\text { atenuación }\end{array}$ & + & $\mathrm{O}$ & + & + & $\mathrm{O}$ & $\mathrm{o}$ \\
\hline Metáfora & o & + & o & o & + & + \\
\hline Onomatopeya & + & 0 & + & + & $\mathrm{o}$ & $\mathrm{o}$ \\
\hline Paralelismo & 0 & $\mathrm{o}$ & 0 & $\mathrm{o}$ & + & $\mathrm{o}$ \\
\hline Polisíndeton & o & o & o & o & + & 0 \\
\hline Prosopopeya & + & + & + & + & & \\
\hline Sarcasmo & $\mathrm{o}$ & + & 0 & 0 & 0 & $\mathrm{o}$ \\
\hline Símbolo & $\mathrm{o}$ & 0 & + & $\mathrm{o}$ & + & + \\
\hline $\begin{array}{l}\text { Símil o } \\
\text { comparación }\end{array}$ & + & $\mathrm{o}$ & + & + & + & + \\
\hline
\end{tabular}

Presencia: + Ausencia: o 
Tabla 5: Cantidad de figuras usadas para cada una de las actividades de instrucción

\begin{tabular}{|c|c|c|c|c|c|c|}
\hline & \multicolumn{6}{|c|}{ ACTIVIDADES } \\
\hline & $\begin{array}{l}\text { Ensalada } \\
\text { de fábulas }\end{array}$ & $\begin{array}{c}\text { El } \\
\text { cuestionario } \\
\text { disparatado }\end{array}$ & $\begin{array}{l}\text { Fábulas } \\
\text { en clave } \\
\text { obligada }\end{array}$ & $\begin{array}{c}\text { La } \\
\text { chistera } \\
\text { del mago }\end{array}$ & Topograma & Acróstico \\
\hline $\begin{array}{c}\text { CANTIDAD } \\
\text { DE } \\
\text { FIGURAS }\end{array}$ & $\begin{array}{c}7 \\
(2,87 \%)\end{array}$ & $\begin{array}{c}9 \\
(3,69 \%)\end{array}$ & $\begin{array}{c}9 \\
(3,69 \%)\end{array}$ & $\begin{array}{c}9 \\
(3,69 \%)\end{array}$ & $\begin{array}{c}12 \\
(4,92 \%)\end{array}$ & $\begin{array}{c}8 \\
(3,28 \%)\end{array}$ \\
\hline
\end{tabular}

Por último, el número de recursos usados por cada uno de los sujetos de cada nivel por géneros (prosa y verso) queda expuesto en las tablas 6,7 y 8 , que permiten comprobar cómo es considerablemente mayor la presencia de recursos en los textos en verso y que, igual que muestran las tablas anteriores, conforme se avanza en los niveles aumenta su aparición.

Tabla 6: Registro del número de recursos utilizados por los sujetos del nivel A2/A2+

\begin{tabular}{|c|c|c|c|}
\hline $\begin{array}{c}\text { SUJETOS } \\
\text { NIVEL } \\
\text { A2/A2+ }\end{array}$ & $\begin{array}{c}\text { COMPOSICIÓN } \\
\text { ESCRITA:PROSA }\end{array}$ & $\begin{array}{c}\text { COMPOSICIÓN } \\
\text { ESCRITA:VERSO }\end{array}$ & TOTAL \\
\hline 1A & 0 & 2 & 2 \\
\hline 1B & 0 & 3 & 3 \\
\hline 1C & 0 & 3 & 3 \\
\hline 1D & 0 & 3 & 3 \\
\hline 1E & 1 & 3 & 4 \\
\hline 1F & 1 & 3 & 4 \\
\hline 1G & 2 & 3 & 5 \\
\hline $1 \mathrm{H}$ & 2 & 4 & 6 \\
\hline $1 \mathrm{I}$ & 1 & 5 & 6 \\
\hline
\end{tabular}

Tabla 7: Registro del número de recursos utilizados por los sujetos del nivel B1.

\begin{tabular}{|c|c|c|c|}
\hline $\begin{array}{c}\text { SUJETOS } \\
\text { NIVEL B1 }\end{array}$ & $\begin{array}{c}\text { COMPOSICIÓN } \\
\text { ESCRITA:PROSA }\end{array}$ & $\begin{array}{c}\text { COMPOSICIÓN } \\
\text { ESCRITA:VERSO }\end{array}$ & TOTAL \\
\hline 2A & 1 & 4 & 5 \\
\hline 2B & 1 & 4 & 5 \\
\hline 2C & 1 & 4 & 5 \\
\hline 2D & 1 & 5 & 6 \\
\hline 2E & 2 & 5 & 7 \\
\hline 2F & 2 & 5 & 7 \\
\hline 2G & 3 & 5 & 8 \\
\hline 2H & 3 & 5 & 8 \\
\hline 2I & 2 & 6 & 8 \\
\hline 2J & 2 & 6 & 8 \\
\hline
\end{tabular}


Tabla 8: Registro del número de recursos utilizados por los sujetos del nivel B1+

\begin{tabular}{|c|c|c|c|}
\hline $\begin{array}{c}\text { SUJETOS } \\
\text { NIVEL B1+ }\end{array}$ & $\begin{array}{c}\text { COMPOSICIÓN } \\
\text { ESCRITA:PROSA }\end{array}$ & $\begin{array}{c}\text { COMPOSICIÓN } \\
\text { ESCRITA:VERSO }\end{array}$ & TOTAL \\
\hline 3A & 3 & 5 & 8 \\
\hline 3B & 3 & 7 & 10 \\
\hline 3C & 3 & 7 & 10 \\
\hline 3D & 2 & 8 & 10 \\
\hline 3E & 4 & 8 & 12 \\
\hline 3F & 3 & 9 & 12 \\
\hline 3G & 4 & 10 & 14 \\
\hline 3H & 4 & 10 & 14 \\
\hline 3I & 4 & 11 & 15 \\
\hline 3J & 5 & 11 & 16 \\
\hline
\end{tabular}

En cifras:

a) Recursos literarios y niveles educativos: se observa una diferencia de 18 puntos en el número de figuras usadas entre los niveles inicial y B1; además, se aprecia un despegue evidente desde el grupo de seconde (14) al de terminale (22).

b) Recursos literarios y grupos: El gráfico número 2 confirma la tendencia visible en el anterior, pues al considerar los niveles en relación con los tres grupos en que se han estructurado los sujetos del estudio, la diferencia entre el grupo 1 y el 3 es de 13 puntos, lo cual corresponde a un aumento porcentual del 5,33\% en la presencia de recursos estilísticos.

c) Recursos literarios y actividades: la relación entre el número de figuras utilizadas en las composiciones escritas y las actividades utilizadas en el periodo de instrucción muestra que son las Fábulas... y el Topograma las que más han incluido $(10,12)$. Además, desde la perspectiva del género literario (prosa o verso) marcan la mayor presencia de los recursos en los textos escritos en verso para todos los grupos (72\% verso; $28 \%$ prosa).

d) Nivel competencial correspondiente a cada uno de los tres grupos considerados: A2/ A2+: 34 poesía, 9 prosa; B1: 53 poesía, 20 prosa; B1+: 87 poesía, 35 prosa. En porcentajes: A2/A2+: $79 \%$ poesía, 21\% prosa; B1: 73\% poesía, 27\% prosa; B1+: $71 \%$ poesía, $29 \%$ prosa.

\section{AnÁlisis y discusión de los Resultados}

El análisis de los principales resultados obtenidos puede sintetizarse como sigue.

Uno. La variedad en el uso de los recursos literarios aumenta conforme aumenta el nivel competencial de los sujetos.

Dos. No es relevante la especificidad de las actividades propuestas para los Textos de Composición Escrita en Prosa (TCEPr). Sí es relevante la especifidad de la actividad TO frente a AC para los Textos de Composición Escrita Poesía (TCEPo). Sí es relevante el conjunto 
de las actividades propuestas para TCEPo frente al conjunto de las propuestas para TCEPr. Con todo, podría prescindirse sin mayor trascendencia de las propuestas EN y AC.

Tres. El aumento del nivel competencial de los sujetos no conlleva una diferenciación significativa en el uso de los recursos por parte de los sujetos de la muestra en los TCEPr. Es decir, la homogeneidad de los distintos grupos de nivel en la utilización de los recursos se mantiene para los mencionados TCEPr. Sin embargo, dicha homogeneidad entre los sujetos de la muestra se rompe para los TCEPo en el grupo B1+. Estos resultados coinciden con los obtenidos por Fernández de Haro, Núñez y Romero (2008) sobre la pauta de la variable que ellos llaman estilo.

Cuatro. Se observa un incremento en la diferencia numérica resultante de la resta del número de recursos utilizados en TCEPo menos TCEPr conforme aumenta el nivel competencial del alumnado; es decir, a mayor nivel competencial, aumenta el número de recursos utilizados en TCEPo frente al número de recursos utilizados en TCEPr.

Cinco. El registro porcentual correspondiente a los recursos utilizados en TCEPo menos TCEPr es inverso respecto a la cuantificación numérica: a mayor nivel, menos diferencia porcentual existe en la utilización de los recursos entre TCEPo y TCEPr. Es decir, conforme aumenta el nivel competencial de los sujetos, el incremento porcentual de los recursos utilizados en TCEPr crece frente al incremento porcentual de los recursos utilizados en TCEPo.

Seis. Los resultados relativos a las repercusiones de las actividades de composición escrita sobre la evolución del dominio de las propiedades textuales apuntan en el mismo sentido de los obtenidos por Rodríguez Polo (2000): el cuidado por el estilo solo aparece una vez que el escritor posee un cierto dominio de, este orden, la coherencia, la cohesión, la corrección y la adecuación. Parece, como también encontraron Romero y Ruiz Ortega (2001), que los escritores noveles, aunque estén produciendo lo que Cassany (1989) denomina "prosa de escritor", no se aventuran a utilizar recursos creativos (estilísticos o al menos pseudoliterarios) si no se sienten seguros de su manejo de la lengua.

\section{Principales conclusiones}

En la adquisición de la competencia comunicativa en la enseñanza del español como lengua extranjera (ELE), los recursos estilísticos amplifican su presencia progresivamente a medida que se avanza en los diferentes niveles, o sea, en el proceso de adquisición de la lengua. Nuestra investigación muestra que se requiere un cierto nivel de dominio de la lengua meta para que se registre la aparición de estos recursos que, tal y como se caracterizan habitualmente, responden a usos de la lengua más allá de lo meramente denotativo, es decir, hasta que no se alcanza un cierto nivel de logro en el dominio de la coherencia, la cohesión, la adecuación y la corrección no aparecen recursos estilísticos o, dicho de otra forma, no hay voluntad de estilo en los aprendices.

Por este motivo, los recursos estilísticos pueden ser considerados como descriptores válidos para precisar la gradación de los niveles A2/B1+ de español lengua extranjera.

En este sentido, y como prospectiva de continuación a tenor de las conclusiones aquí explicitadas, se propone la elaboración de un test para su evaluación y validación ecológica teniendo en cuenta, como datos cruzados, el tipo de recurso, el tipo de estrategia y el nivel competencial del sujeto, de modo que se pueda profundizar en los factores que intervienen en el uso de tales recursos. 


\section{REFERENCIAS BIBLIOGRÁFICAS}

Alarcón Gómez, E. (2011). "Diagnosis de un programa de intervención para el desarrollo de la composición escrita", en Alhucema. Revista Internacional de Teatro y Literatura, 26: 85-96.

Alvarado, M., Rodríguez M.C. y Tobelem, M. (1981). GRAFEIN: Teoría y práctica de un taller de escritura. Madrid: Altalena.

Ausubel, D. (1968). Educational Psychology: A Cognitive view. Nueva York: Holt.

Blas Aritio, F. de A. (2008). "La formación profesional basada en la competencia", en Avances en Supervisión Educativa. Revista de la Asociación de Inspectores de Educación de España, 9: 68-76.

Bronckart, J.P. (2008). “Es pertinente la noción de competencia en la educación?”, en Novedades Educativas, vol. 211: 4-12.

Brooks, N. (1966). "Language teaching: the new approach", in Phi Delta Kappan 47:357-9.

Canale, M., Swain, M. (1996). "Fundamentos teóricos de los enfoques comunicativos", en Signos 17 y $18: 54-62$ y $78-89$.

Cassany, D. (1989). Describir el escribir. Barcelona: Paidós.

Cassany, D. (1993). Reparar la escritura. Didáctica de la corrección de lo escrito. Barcelona: Paidós.

Cassany, D. (1995). La cocina de la escritura. Barcelona: Anagrama.

Consejo de Europa (2002). Marco común europeo de referencia para las lenguas: aprendizaje, enseñanza y evaluación. Madrid: Instituto Cervantes.

Dendelauce, I. (1994). "Diseños cuasiexperimentales", en V. García Hoz (dir.). Problemas y métodos de investigación en educación personalizada. Madrid: Rialp.

Fernández de Haro, E., Núñez, M.P. y Romero, A. (2009). “Adquisición de habilidades metalingüísticas y enseñanza-aprendizaje de la composición escrita en educación primaria: reflexiones didácticas sobre los resultados de un estudio experimental", en Porta linguarum, 12, 149-16.

Fleming, M. (2006). "The use and mis-use of competence framaworks and statements with particular attention to describing achievement in literature", in Towards a Common European Framework of Reference for Languages of School Education? Selected conference papers. Ed. W. Martyniuk. Council of Europe. Language Policy Division and the Jagiellonian University, 47-59.

Freinet, C. (1973). El texto libre. Barcelona: Laia.

García Rivera, G. (1995). Didáctica de la literatura para la Enseñanza Primaria y Secundaria. Madrid: Akal.

González Piñeiro, M., Guillén Díaz, C., Vez, J.M. (2010). Didáctica de las lenguas modernas: competencia plurilingüe e intercultural. Madrid: Síntesis.

Flower, L. \& Hayes, J. R. (1980). "Identifying the Organization of Writing Processes", in L.W. Gregg \& E.R. Steinberg (comps.). Cognitive Processes in Writing, pp. 3-30. New Jersey: Erlbaum.

Johnson, R.K. (1989). The Second Language Curriculum. Cambridge: Cambridge University Press.

Madrid, D. y N. McLaren (1995). Didactic Procedures for TEFL. Valladolid: La Calesa.

Madrid, D. (1997). "La evaluación del área curricular de la lengua extranjera", en H. Salmerón (ed.). Evaluación educativa: Teoría, metodología y aplicaciones en áreas de conocimiento. Granada: Grupo Editorial Universitario, 252-290.

Mendoza Fillola, A (1999). "El tratamiento didáctico de la literatura desde una perspectiva intertextual", en A. Romero y otros (eds.): Educación lingüistica y literaria en el ámbito escolar. Granada: GEU. 
Navarro, J.M. (1992). "La enseñanza de la literatura en el D.C.B.”, en P. Cerrillo y J. García Padrino (coords.). Literatura infantil y enseñanza de la literatura. Cuenca: Universidad de Castilla-La Mancha.

Núñez Delgado, P. (2003). Didáctica de la comunicación oral: Bases teóricas y orientaciones metodológicas para el desarrollo de la competencia discursiva oral en la educación obligatoria. Granada: GEU.

Núñez Delgado, P. y Romero López, A. (2003). Investigación en didáctica de la lengua e innovación curricular. Granada: Universidad de Granada.

Olsbu, I., Salkjelsvik, K.S. (2006). "La literatura en clase: Leer hacia la competencia literaria". I Congreso Nacional 'ANPE: 2006, año español en Noruega: un reto posible'. ANPE.

Rienda, J. (2006a). Cuaderno didáctico para el libro "El buen amigo”. Granada: Port-Royal.

Rienda, J. (2006b). Cuaderno de composición escrita. Granada: Port-Royal.

Rienda, J. (2010). Nociones elementales de didáctica de la literatura. Granada: Alhulia.

Rivas, E. (1991). Metodología de la investigación científica. Trujillo: Centro Psicopedagógico Libertad.

Rivers, W. M. (1968/1981). Teaching Foreign-Language Skills. Chicago and London: University of Chicago Press.

Rodari, G. (1983). Gramática de la fantasía. Introducción al arte de inventar historias. Buenos Aires: Colihue.

Romero, A. y Ruiz Ortega, F. (2001). Acercamiento al texto poético: un programa de intervención didáctica para la educación primaria. Granada: GEU.

Samoilovich, D. (1979). Cómo jugar y divertirse con escritores. Madrid: Altalena.

Sánchez Pérez, A. (1993). Hacia un método integral de la enseñanza de idiomas. Madrid: SGEL.

Tuxworth, E., (1989). "Competence based education and training: background and origins", in J.W. Burke (Ed.). Competence based education and training. London: Falmer Press, 10-25.

Wilkins D.A. (1976). Notional Syllabuses. London: Oxford University Press. 